Discrete Comput Geom 32:471-479 (2004)

DOI: $10.1007 / \mathrm{s} 00454-004-1134-2$

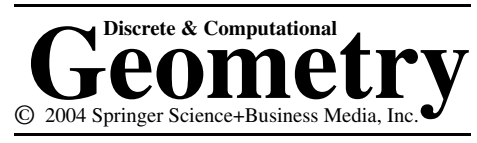

\title{
A Complexity Bound on Faces of the Hull Complex*
}

\author{
Mike Develin \\ American Institute of Mathematics, 360 Portage Ave., \\ Palo Alto, CA 94306-2244, USA \\ develin@math.berkeley.edu
}

\begin{abstract}
Given a monomial $k\left[x_{1}, \ldots, x_{n}\right]$-module $M$ in the Laurent polynomial ring $k\left[x_{1}^{ \pm 1}, \ldots, x_{n}^{ \pm 1}\right]$, the hull complex is defined to be the set of bounded faces of the convex hull of the points $\left\{t^{a} \mid x^{a} \in M\right\}$ for sufficiently large $t$. Bayer and Sturmfels [2] conjectured that the faces of this polyhedron are of bounded complexity in the sense that every such face is affinely isomorphic to a subpolytope of the $(n-1)$-dimensional permutohedron, which in particular would imply that these faces have at most $n$ ! vertices. In this paper we prove that the latter statement is true, and give a counterexample to the stronger conjecture.
\end{abstract}

\section{Introduction}

The hull complex, an object defined by Bayer and Sturmfels [2], is an important object yielding a canonical free resolution of a monomial module. The significance of this resolution in commutative algebra is explained in the Appendix; however, we are concerned here with a combinatorial conjecture in [2], and our methods are purely combinatorial. To that end, we have decided to present the combinatorial setup free from the technical algebraic background which spawned the question; readers interested in learning the motivation should consult the Appendix.

Consider the Laurent polynomial $\operatorname{ring} T=k\left[x_{1}^{ \pm 1}, \ldots, x_{n}^{ \pm 1}\right]$, which contains the polynomial ring $S=k\left[x_{1}, \ldots, x_{n}\right]$. A monomial module is defined to be an $S$-submodule of $T$ which is generated by monomials $x^{\mathbf{a}}=x_{1}^{a_{1}} \cdots x_{n}^{a_{n}}$. The hull complex of a monomial module $M$ with no infinite decreasing sequence of monomials (under divisibility) is defined to be the set of bounded faces of the convex hull of the points $\left\{t^{\mathrm{a}} \mid x^{\mathrm{a}} \in M\right\}$, where $t$ is sufficiently large and $t^{\mathbf{a}}:=\left(t^{a_{1}}, \ldots, t^{a_{n}}\right)$. This polyhedron $P_{t}$ has fixed combinatorial type for $t>(n+1)$ !. If $M$ is generated by $x^{\mathbf{v}_{1}}, \ldots, x^{\mathbf{v}_{r}}$, then $P_{t}$ is expressible as $\operatorname{conv}\left(\left\{t^{\mathbf{v}_{1}}, \ldots, t^{\mathbf{v}_{r}}\right\}\right)+\mathbb{R}_{+}^{n}$, where $\mathbb{R}_{+}^{n}$ is the positive orthant.

* The author was supported during this work by an NSF Graduate Research Fellowship. 
In general, if $Q$ is any polytope, we can calculate the bounded faces of $P:=Q+\mathbb{R}_{+}^{n}$ as follows. First, they are clearly a subset of the faces of $Q$. For each face $F$ in $Q$, take a face-defining linear functional $\omega$ of $F$, which is of the form $\sum c_{i} y_{i}$, with this sum being minimized (over $Q$ ) on $F$. Then it is easy to see that $F$ will be the face of $P$ defined by $\omega$ if and only if the $c_{i}$ are all positive. If they are all positive, then $\omega(y+z) \geq \omega(y)$, where $y \in Q$ and $z \in \mathbb{R}_{+}^{n}$; this will be minimized precisely when $z=0$ and $y \in F$, i.e., when $y+z \in F$.

Conversely, if some $c_{i}$ is equal to 0 or negative, then clearly $\omega$ cannot define a bounded face of $P$, as it does not even attain a minimum value. So, when determining if a face $F$ of $Q$ is a bounded face of $P$, it suffices to check if there exists a face-defining linear functional of $F$ with all positive coefficients.

Example 1. Let $n=3$, and let $M$ be the monomial module generated by the four monomials $\left\{x_{1}^{2}, x_{2}^{2}, x_{3}^{2}, x_{1} x_{2} x_{3}\right\}$.

In this case the polyhedron $P_{t}$ has vertices $v_{1}=\left(t^{2}, 1,1\right), v_{2}=\left(1, t^{2}, 1\right), v_{3}=$ $\left(1,1, t^{2}\right)$, and $v_{4}=(t, t, t)$; it is conv $\left(\left\{v_{1}, v_{2}, v_{3}, v_{4}\right\}\right)+\mathbb{R}_{+}^{n}$. For $t$ large, the bounded faces of this form a simplicial complex with maximal faces $\left\{v_{1}, v_{2}, v_{4}\right\},\left\{v_{1}, v_{3}, v_{4}\right\}$, and $\left\{v_{2}, v_{3}, v_{4}\right\} ;\left\{v_{1}, v_{2}, v_{3}\right\}$ has facet-defining inequality $-1\left(y_{1}\right)-1\left(y_{2}\right)-1\left(y_{3}\right) \geq-2-t^{2}$, and thus is not a face of $P_{t}$. This polyhedron (for sufficiently large $t$ ) is depicted in Fig. 1 .

Bayer and Sturmfels made the following conjecture about the faces of the hull complex, where a subpolytope refers to a polytope which is the convex hull of a subset of the vertices of the original polytope.

Conjecture 1. Every face of hull(M) is affinely isomorphic to a subpolytope of the $(n-1)$-dimensional permutohedron and hence has at most $n$ ! vertices.

The $(n-1)$-dimensional permutohedron is defined to be the convex hull of $\{(\pi(1), \ldots, \pi(n))\}$ in $\mathbb{R}^{n}$, where $\pi$ ranges over all permutations in $S_{n}$. We can generalize

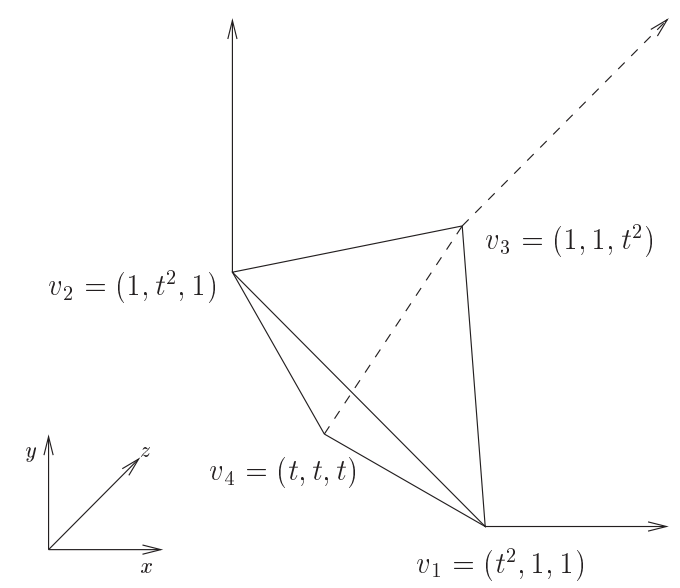

Fig. 1. The polyhedron $P_{t}$ corresponding to the ideal $\left(x_{1}^{2}, x_{2}^{2}, x_{3}^{2}, x_{1} x_{2} x_{3}\right)$. 
this slightly by considering all $(n-1)$-dimensional permutohedra; such a permutohedron is defined as the convex hull of the points $\left\{\left(u_{\pi(1)}, \ldots, u_{\pi(n)}\right)\right\}$, where $\left(u_{1}, \ldots, u_{n}\right)$ is an $n$-tuple of distinct real numbers, and $\pi$ ranges over all permutations in $S_{n}$. In Section 2 we construct a counterexample where the hull complex has a face which is not even combinatorially isomorphic to any subpolytope of any such permutohedron.

While this conjecture is false, it is true that the faces of the hull complex have bounded complexity; in particular, in Section 3 we prove that every face of hull $(M)$ has at most $n$ ! vertices.

\section{Counterexample to the Permutohedron Conjecture}

In this section we construct two counterexamples to Conjecture 1 . The first is a fairly simple example; the second shows that there exist faces of the hull complex which are not even combinatorially isomorphic to any subpolytope of a permutohedron.

First, let $n=3$, so that $S=k\left[x_{1}, x_{2}, x_{3}\right]$ and $T=k\left[x_{1}^{ \pm 1}, x_{2}^{ \pm 1}, x_{3}^{ \pm 1}\right]$. Let $M$ be the monomial module generated by $x^{\mathbf{a}}$, where a ranges over the columns of

$$
\left(\begin{array}{llllll}
5 & 5 & 3 & 2 & 4 & 2 \\
2 & 1 & 5 & 5 & 1 & 4 \\
0 & 2 & 0 & 3 & 5 & 5
\end{array}\right) .
$$

We claim that the six points $t^{\text {a }}$ corresponding to these generators form a facet for $t$ sufficiently large.

Indeed, consider the linear functional $f=\left(t^{2}+t+1\right) y_{1}+\left(t^{2}+t\right) y_{2}+\left(t^{2}\right) y_{3}$ on the space where $P_{t}$ resides. This linear functional evaluates to $t^{7}+t^{6}+t^{5}+t^{4}+t^{3}+t^{2}$ on all six of the generators, and because each coefficient is positive this is the minimal value of $f$ on the points $\mathbf{y}=t^{\mathbf{a}}$ for $x^{\mathbf{a}}$ a monomial in $M$, and hence these six points comprise the facet $G$ of $P_{t}$ defined by $f$.

However, this hexagon is not affinely isomorphic to any subpolytope of any permutohedron $\Pi_{2} . \Pi_{2}$ has six vertices, so if such a subpolytope exists it must be all of $\Pi_{2}$. In both $\Pi_{2}$ and $G$, pairs of opposite sides are parallel; consider the ratios between the lengths of the three pairs of opposite sides. Each of these ratios is of course preserved under affine isomorphism. In $\Pi_{2}$ it is easy to check that all three ratios are the same, and equal to $\left(u_{3}-u_{2}\right) /\left(u_{2}-u_{1}\right)$, where $u_{1}<u_{2}<u_{3}$ are the three numbers defining the permutohedron. On the other hand, in $G$ these ratios are equal to $t^{3}, t^{2}$, and $t$, and thus the two hexagons cannot be affinely isomorphic.

This face of the hull complex is, however, combinatorially isomorphic to the permutohedron itself, so one might ask whether or not all faces of the hull complex have the same combinatorial type as a subpolytope of the permutohedron. Extending this example to four dimensions, however, shows that this cannot be the case. Here we have $n=4$, and $M$ is the monomial module generated by $x^{\mathbf{a}}$ where a ranges over the columns of

$$
\left(\begin{array}{llllllllllllllllllllllll}
9 & 9 & 9 & 9 & 9 & 9 & 6 & 6 & 4 & 3 & 5 & 3 & 7 & 7 & 4 & 3 & 6 & 3 & 8 & 8 & 5 & 3 & 6 & 3 \\
5 & 5 & 3 & 2 & 4 & 2 & 9 & 9 & 9 & 9 & 9 & 9 & 3 & 2 & 7 & 7 & 2 & 6 & 4 & 2 & 8 & 8 & 2 & 6 \\
2 & 1 & 5 & 5 & 1 & 4 & 2 & 1 & 6 & 6 & 1 & 5 & 9 & 9 & 9 & 9 & 9 & 9 & 1 & 4 & 1 & 5 & 8 & 8 \\
0 & 2 & 0 & 3 & 5 & 5 & 0 & 2 & 0 & 4 & 6 & 6 & 0 & 3 & 0 & 4 & 7 & 7 & 9 & 9 & 9 & 9 & 9 & 9
\end{array}\right) .
$$




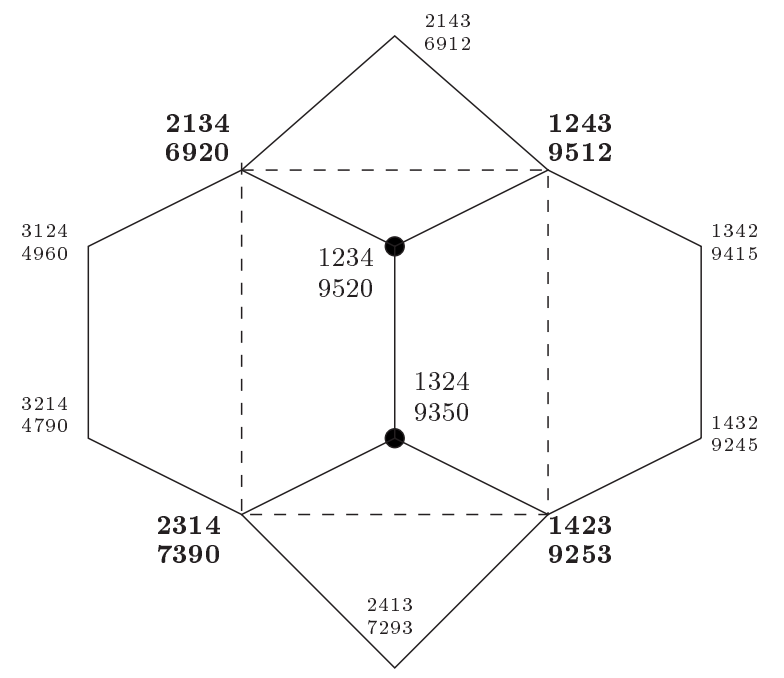

Fig. 2. Part of the permutohedron.

As before, the linear functional $\left(t^{3}+t^{2}+t+1\right) y_{1}+\left(t^{3}+t^{2}+t\right) y_{2}+\left(t^{3}+t^{2}\right) y_{3}+\left(t^{3}\right) y_{4}$ demonstrates that the 24 elements $t^{\text {a }}$ corresponding to these 24 generators lie on a facet of the hull complex of $M$, and in fact this facet is easily checked to be combinatorially isomorphic to $\Pi_{3}$ via the obvious map between vertices. However, we claim that if one removes the generators corresponding to $(9,5,2,0)$ and $(9,3,5,0)$, the resulting polytope is not even combinatorially isomorphic to any subpolytope of $\Pi_{3}$.

Indeed, consider Fig. 2, which shows a portion of the (combinatorially defined) permutohedron, and the labels that are mapped to this from both the standard permutohedron and this object. The top labels correspond to the standard construction of the permutohedron; the bottom labels correspond to the generators of $M$ which form its vertices in the hull complex. The four bold vertices are coplanar in the standard construction, but not in the hull complex.

If we remove the two vertices corresponding to these generators, the four vertices in bold all lie in the same plane in the standard permutohedron, and in fact in any permutohedron; the resulting object then has an additional square facet. However, these four vertices do not lie in the same plane in the hull complex of $M$, and consequently when these two vertices are removed from the hull complex, the resulting object is a mutated permutohedron with two additional triangular facets in place of the square facet. Indeed, it is easy to check that one cannot remove two vertices anywhere from a permutohedron and obtain this combinatorial polytope.

Consequently, if we consider the monomial module $M^{\prime}$ generated by the 22 other generators of $M$, one of its facets will contain all 22 generators (using the same linear functional), and will not be combinatorially isomorphic to any subpolytope of any permutohedron. 


\section{Faces of hull $(M)$ Have at Most $n$ ! Vertices}

In this section we show that for fixed $n$, the complexity of a face of the hull complex is bounded independent of the monomial module $M$. To be precise, we prove the following theorem.

Theorem 1. For any monomial module $M \subset k\left[x_{1}^{ \pm 1}, \ldots, x_{n}^{ \pm n}\right]$, each face of the hull complex hull $(M)$ has at most $n$ ! vertices.

Proof. We first consider the case where the face $F$ in question is a facet, i.e., is $(n-1)$ dimensional. If there are only $n$ vertices, there is nothing to prove. If not, let $H$ be the hyperplane whose intersection with the polytope $P_{t}$ is $F$, and let $t^{\mathbf{a}_{1}}, \ldots, t^{\mathbf{a}_{n+1}}$ be vertices of $F$ whose affine span is $H$. Given any set of vectors $x_{i}$, the dimension of the linear span of the vectors $\left(x_{i}, 1\right)$ is one greater than the dimension of the affine span of the vectors $x_{i}$. Let $T$ be the matrix whose columns are $\left(t^{\mathrm{a}_{i}}, 1\right)^{\mathrm{T}}$; this matrix is therefore of rank $n$. These statements hold for all sufficiently large $t$, and so the matrix $T$ has determinant 0 for infinitely many values of $t$. Therefore, its determinant must be 0 as a polynomial in $t$.

Since the $n+1$ rows of this matrix do not have full rank, there exists a linear combination of them with coefficients in $\mathbb{Q}(t)$ that is equal to 0 . Multiplying through by the common denominator, this means that there exist polynomials $f_{1}, \ldots, f_{n}, g \in \mathbb{Q}[t]$ such that for each $i$ we have

$$
f_{1} t^{a_{i 1}}+f_{2} t^{a_{i 2}}+\cdots+f_{n} t^{a_{i n}}=g .
$$

Now, because $F$ is of codimension 1, there exists a unique hyperplane containing $F$, and thus a unique (up to scalar multiplication) linear equality valid on $F$. On the other hand, (1) is of this form, and so (possibly after multiplying by -1 ) the facet-defining inequality of $F$ up to a positive constant must be

$$
f_{1} y_{1}+f_{2} y_{2}+\cdots+f_{n} y_{i} \geq g
$$

We claim that each $f_{i}$ must have a positive leading coefficient; consider without loss of generality $f_{1}$. By construction, $x_{1}^{a_{i 1}} x_{2}^{a_{i 2}} \cdots x_{n}^{a_{i n}} \in M$, and since $M$ is an $S$-module this means that $x_{1}^{a_{i 1}+1} x_{2}^{a_{i 2}} \cdots x_{n}^{a_{i n}}$ must be as well. Therefore, $\left(t^{a_{i 1}+1}, t^{a_{i 2}}, \ldots, t^{a_{i n}}\right) \in P_{t}$. However, if the leading term of $f_{1}$ is negative, for large enough $t, f_{1}$ will also be negative, and this point will then violate (2). Similarly, if $f_{1}=0$, then adding any constant to $a_{i 1}$ will yield a point on $F$, and thus $F$ will necessarily be unbounded.

Now, to complete the proof in the case where $F$ is a facet, we define a map from the set of $\left(a^{i 1}, \ldots, a^{i n}\right)$ which yield equality in (1) to the set of orderings of $\{1,2, \ldots, n\}$. Let the degree of $f_{j}$ be $b_{j}$, and let the degree of $g$ be $d$. Consider the set of all $j$ for which $b_{j}+a_{i j}=d$, and write down these $j$ (in any order) inside a set of parentheses. Now, eliminate the terms on the left side corresponding to these $j$, and subtract the corresponding sum from $g$; what is left is an equation of the same form as (1), with fewer terms. Since the terms are a subset of the original terms, the condition that the $f_{i}$ 
still have positive leading coefficients still holds. Iterate this process until all terms are gone and we are left with $0=0$, so that we have something like $(583)(6)(7)(14)(2)$.

Because the leading coefficient of each $f_{i}$ is positive, it follows immediately that given a string of numbers, there is at most one way to insert the parentheses to yield an acceptable expression; given a parenthesized expression, it is equally clear that the $a_{i j}$ are uniquely determined (we can read off the top group as $d-b_{j}$ for each $j$ in it, and proceed inductively). Consequently, for each ordering of the numbers $1,2, \ldots, n$ there is at most one corresponding point in $H \cap P_{t}$. This yields $n$ ! as an upper bound on the number of vertices of $F$.

Next, suppose that $F$ is not a facet. For each (possibly unbounded) facet of $P_{t}$ containing $F$, pick points which affinely span it. Each facet therefore yields an equality condition as in (1), except that the $f_{i}$ may be 0 . All nonzero $f_{i}$ must still have positive leading coefficients, and as before we obtain (2) as a valid inequality on $P_{t}$.

However, any face of a polyhedron is the intersection of the facets containing it; consequently, we can add the facet-defining inequalities to obtain an inequality that defines the face $F$. This inequality must have each $f_{i}$ either equal to 0 or with a positive leading term, since each of its summands does; furthermore, no $f_{i}$ can be equal to 0 , since as before this would yield that $F$ is unbounded. From here, we can enumerate the points as in the remainder of the proof in the facet case to yield that $F$ has at most $n$ ! vertices as desired.

\section{Future Questions}

We have proven that the stronger conjecture of Bayer and Sturmfels is false, but that the basic idea that the complexity of the faces of the hull complex is limited is true. There is quite a bit of middle ground; the example we constructed in Section 2 has an obvious extension to higher dimensions, and each of these has the same combinatorial type as the permutohedron. Is it true that every face of the hull complex is combinatorially isomorphic to some subpolytope of a combinatorial permutohedron? If so, is there a nice characterization of polytopes which can be represented as subpolytopes of combinatorial permutohedra?

The map we constructed in Section 3, from the vertices of a face of the hull polytope to the orderings of $\{1,2, \ldots, n\}$, yields in a natural way a face of the permutohedron $\Pi_{n-1}$ for each vertex. In particular, the faces of the permutohedron can be indexed by parenthesized sequences of the form (583)(6)(7)(14)(2) [5], taking the parentheses into consideration; for instance, this sequence corresponds to the face which has 6,7 , and 8 (in some order) in coordinates 5,8, and 3, 5 in coordinate 6, 4 in coordinate 7, 2 and 3 in coordinates 1 and 4 , and 1 in coordinate 2. Our map is similar; the parenthesized sequence above, up to normalization, says that for this vertex, variables 5, 8, and 3 have the largest exponent (or that those coordinates have the largest values), followed by variable 6 , then variable 7 , then variables 1 and 4 , and finally variable 2 .

Does this map yield useful information about the faces of the permutohedron? The exact nature of the connection between faces of the hull complex and permutohedra is still mysterious. How can one describe the faces of the permutohedron using this language? 
What can we say about the hull complex itself independent of the information about the faces? Can we bound the number of vertices in a face of dimension $k$, say by $k$ !?

\section{Acknowledgment}

I thank Bernd Sturmfels for bringing this conjecture to my attention.

\section{Appendix. Commutative Algebra Background}

The material in this appendix was compiled with help from the forthcoming book Combinatorial Commutative Algebra, by Miller and Sturmfels [3].

As usual, let $T$ be the Laurent polynomial ring $k\left[x_{1}^{ \pm 1}, \ldots, x_{n}^{ \pm 1}\right]$, which contains the polynomial ring $S=k\left[x_{1}, \ldots, x_{n}\right]$. Recall that a monomial module is an $S$-submodule of $T$ which is generated by monomials $x^{\mathbf{a}}=x_{1}^{a_{1}} \cdots x_{n}^{a_{n}}$.

An important construction in commutative algebra, which can be applied to monomial modules, is that of the free resolution. This is an exact sequence, where the kernel of one map is equal to the image of the previous one, of the form

$$
0 \rightarrow \bigoplus S\left[-\mathbf{a}_{n j}\right] \cdots \bigoplus S\left[-\mathbf{a}_{2 j}\right] \rightarrow \bigoplus S\left[-\mathbf{a}_{1 j}\right] \rightarrow M \rightarrow 0 .
$$

Each $S\left[-\mathbf{a}_{n j}\right]$ is a copy of $S$, shifted in degree; if $\mathbf{d}$ is a multidegree, then the $\mathbf{e} t h$ graded part of $S[\mathbf{d}]$ is defined to be $S_{\mathbf{d}+\mathbf{e}}$. This relabeling of degree is done so that each map is homogeneous of degree 0 in the natural $\mathbb{Z}^{n}$-grading on each part of the map.

From such a free resolution, we can obtain many useful facts about the module $M$, and in the case where $M$ is an ideal about the variety corresponding to $M$. The game is most afoot when the free resolution is minimal in the sense that the number of copies of $S$ in each $\oplus S\left[-\mathbf{a}_{i j}\right]$, for fixed $i$, is simultaneously minimized. In this case, for instance, the $\mathbf{a}_{i j}$ comprise the Betti numbers of $M$, invariants with algebro-geometric connections to the variety $V(M)$. Explicitly computing these minimal resolutions, however, is in general a difficult problem, even for monomial ideals.

One method of attacking this problem is via cellular complexes. Consider for ease of description a finitely generated monomial module $M$, with minimal generating set $\min (M)=\left\{m_{j}=x^{\mathbf{a}_{j}} \mid j \in I\right\}$. Now, consider any regular cell complex $X$ with $I$ as its set of vertices, and equipped with an incidence function $\varepsilon\left(F, F^{\prime}\right)$ on pairs of faces such that $\varepsilon\left(F, F^{\prime}\right)=0$ unless $F^{\prime}$ is a facet of $F$. In this case the incidence function prescribes $\varepsilon\left(F, F^{\prime}\right)= \pm 1$ in such a way that for any codimension-2 face $F^{\prime}$ of $F$,

$$
\varepsilon\left(F, F_{1}\right) \varepsilon\left(F_{1}, F^{\prime}\right)+\varepsilon\left(F, F_{2}\right) \varepsilon\left(F_{2}, F^{\prime}\right)=0,
$$

where $F_{1}$ and $F_{2}$ are the two facets of $F$ containing $F^{\prime}$. (There are exactly two; this is the diamond property of the face lattice of a regular cell complex.) In addition, we require that $\varepsilon(j, \varnothing)=1$ for all vertices $j$. For example, one could take the set of faces of any polytope with a vertex set labeled with the elements of $I$.

Given these conditions, we can form a cellular complex $F_{X}$. For each face $F$ in $X$, define the monomial $m_{F}=\operatorname{lcm}\left\{\mathrm{m}_{\mathrm{j}} \mid \mathrm{j} \in \mathrm{F}\right\}$, and call its degree $\mathbf{a}_{F}$. Then define $F_{X}=\bigoplus_{F \in X, F \neq \varnothing} S\left[-\mathbf{a}_{F}\right]$, where the differential takes the generator, labeled $F$, of 
$S\left[-\mathbf{a}_{F}\right]$ to

$$
\sum_{F^{\prime} \in X, F^{\prime} \neq \varnothing} \varepsilon\left(F, F^{\prime}\right) \frac{m_{F}}{m_{F^{\prime}}} F^{\prime} .
$$

The homological degree of each face is its dimension in the cell complex $X$.

For a concrete example, consider the Taylor complex of $M$ [4]. This is the cellular complex associated to the simplicial complex $\Delta$ consisting of all subsets of $I$, the set of minimal generators of $M$, with the incidence function $\varepsilon\left(F, F^{\prime}\right)=(-1)^{j}$, where $j$ is the element removed to obtain the facet $F^{\prime}$ from $F$. The Taylor complex is a natural free resolution of $M$, but in general is far from minimal. We provide an example to illustrate the relatively simple computation of these fairly intimidating objects.

Example 2. Let $M$ be the monomial ideal generated by $x^{\mathbf{a}}$, where a $\in$ $\{(0,1,2),(2,1,0),(2,0,1)\}$.

In this example the relevant monomials associated to the faces are as follows: $m_{\varnothing}=1$, $m_{1}=x^{(0,1,2)}, m_{2}=x^{(2,1,0)}, m_{3}=x^{(2,0,1)}, m_{12}=x^{(2,1,2)}, m_{13}=x^{(2,1,2)}, m_{23}=x^{(2,1,1)}$, and $m_{123}=x^{(2,1,2)}$. The Taylor complex is then

$$
\begin{aligned}
0 & \rightarrow S[-(2,1,2)] \rightarrow S[-(2,1,2)] \oplus S[-(2,1,2)] \oplus S[(-2,1,1)] \\
& \rightarrow S[-(0,1,2)] \oplus S[-(2,1,0)] \oplus S[-(2,0,1)],
\end{aligned}
$$

which resolves $M$ in the sense that appending $\rightarrow M \rightarrow 0$ to the end of the complex with the obvious map makes it an exact sequence. The maps are given by taking $1 \epsilon$ $S[-(2,1,2)]$ to $\left(1,1, x_{3}\right)$, and so on. These maps are homogeneous because of the shift in degree.

The point is that Taylor complexes are easy to write down, and yield free resolutions of $M$. As illustrated above, though, these are far from minimal; the search for a good algorithm to yield a (hopefully natural) minimal free resolution for a monomial module $M$ goes on. Another construction is that of the Scarf complex [1]. This is again a simplicial complex, and its faces consist of the set

$$
\left\{F \subseteq I \mid m_{F} \neq m_{G} \text { for all } G \subseteq I, G \neq F\right\} .
$$

In other words, the Scarf complex consists of those subsets of the minimal generating set whose least common multiple is unique among the set of least common multiples of such subsets.

When $M$ is generic, the Scarf complex is a minimal free resolution of $M$. However, when $M$ is not generic, the Scarf complex is not even a resolution of $M$; for instance, in Example 2 the Scarf complex consists of the Taylor complex without the three $S[-(2,1,2)]$ terms, which is not exact.

With this obstacle in mind, Bayer and Sturmfels [2] defined the hull resolution, a canonical free resolution which is equal to the Scarf resolution (and thus minimal) in the case of a generic monomial module $M$. The hull complex of a monomial module $M$ is the set of bounded faces of the convex hull of the points $\left\{t^{\mathbf{a}} \mid x^{\mathbf{a}} \in M\right\}$, where $t$ is sufficiently large and $t^{\mathbf{a}}:=\left(t^{a_{1}}, \ldots, t^{a_{n}}\right)$; this polyhedron $P_{t}$ has fixed combinatorial type for $t>(n+1)$ ! [2]. This resolution has the benefit of yielding a minimal resolution when 
$M$ is generic while still yielding a resolution in the nongeneric case; the polyhedra $P_{t}$ also form a natural class of polytopes with interesting combinatorial structure. Analysis of this combinatorial structure yields information about the hull resolution.

\section{References}

1. D. Bayer, I. Peeva, and B. Sturmfels, Monomial resolutions, Math. Res. Lett. 5 (1998), 31-46.

2. D. Bayer and B. Sturmfels, Cellular resolutions of monomial modules, J. Reine Angew. Math. 502 (1998), 123-140.

3. E. Miller and B. Sturmfels, Combinatorial Commutative Algebra, Graduate Texts in Mathematics, Springer, New York, to appear.

4. D. Taylor, Ideals Generated by Monomials in an $R$-Sequence, Ph.D. thesis, University of Chicago, 1966.

5. G. Ziegler, Lectures on Polytopes, Graduate Texts in Mathematics, Springer, New York, 1995.

Received May 18, 2003, and in revised form January 18, 2004. Online publication September 13, 2004. 